\title{
SELEÇÃO DE GENÓTIPOS DE FEIJOEIRO À SECA ${ }^{1}$ SELECTION OF BEAN PLAN GENOTYPES
}

\author{
Willian Bucker Moraes ${ }^{2}$; Sebastião Martins Filho ${ }^{5}$; Giovanni de Oliveira Garcia ${ }^{4}$; \\ Simone de Paiva Caetano ${ }^{3}$; Wanderson Bucker Moraes ${ }^{3}$
}

\section{RESUMO}

O presente trabalho teve como objetivo avaliar genótipos de feijoeiro com o propósito de selecionar materiais com capacidade de tolerância à seca. O experimento foi conduzido dentro de casa de vegetação no campus do Centro de Ciências Agrárias da Universidade Federal do Espírito Santo (CCAUFES), Alegre-ES. Devido ao grande número de genótipos a serem avaliados o experimento foi realizado em duas fases, o delineamento utilizado foi o inteiramente casualizados no esquema de parcelas subdivididas, sendo a umidade do solo no período de estresse hídrico determinada num intervalo de dois dias, pelo método EDABO (Evaporação Direta da Água em Banho de Óleo). Em termos de produtividade foi possível verificar a diferença não-significativa entre os sistemas de cultivo sem e com estresse para os genótipos IN 2, IN 9, IN 12, IN 14, IN 16, IN 17, IN 18, EL-22, IAPAR 81, Vermelho e Brinco de Ouro, mostrando, desta forma, que estes genótipos são tolerantes ao estresse hídrico; porém estes materiais, na maioria, foram pouco produtivos. Para o número de flores, característica esta que acarreta menor número de vagens e consequentemente menor produtividade, os genótipos Caeté pé Curto, Iconha, Vagem Riscada e Macuquinho, apresentaram-se suscetíveis ao déficit hídrico. Para os demais genótipos não houve diferença significativa entre os sistemas de cultivo, mostrando que estes genótipos, para esta característica, se comportaram de maneira semelhante, tanto na presença quanto na ausência do estresse hídrico.

Palavras chave: Phaseolus vulgaris L., tolerancia la sequía, déficit de agua.

\section{ABSTRACT}

The present work had as objective evaluates bean plant genotypes with the purpose of selecting materials with capacity of tolerance the drought. The experiment was driven inside of greenhouse in the campus of the Center of Agrarian Sciences of Espirito Saint Federal University (CCAUFES), Alegre-ES. Due to the great number of genotypes to they be appraised the experiment was accomplished in two stages, the used delineate was it entirely casualty in the outline of subdivided portions, being the humidity of the soil in the period of water déficit in interval of two days, for method EDABO (Direct Evaporation of Water in Bath of Oil). In productivity terms it was possible to verify the no-significant difference among the cultivation systems without and with stress for the genotypes IN 2, IN 9, IN 12, IN 14, IN 16, IN 17, IN 18, EL-22, IAPAR 81, Red and Earring of Gold, showing, this way, that these genotypes are tolerant to water deficit; however these materials, in most, were little productive. For the number of flowers, characteristic this that carts smaller number of husk and consequently smaller productivity, the genotypes Caeté pé curto, Iconha, Vagem riscada and Macuquinho, came susceptible to the water deficit. For the other genotypes there was not significant difference among the cultivation systems, showing that these genotypes, for this characteristic, behaved in a similar way, in the presence and in the absence of the water déficit.

Key words: Phaseolus vulgaris $L$., drought tolerance, water deficit.

\section{INTRODUÇÃO}

O feijoeiro (Phaseolus vulgaris L.) é um alimento básico para o brasileiro, chegando a representar um componente quase que obrigatório da dieta da população rural e urbana. Yokoyama (2002) cita que o consumo per capita de feijão é de 13,8 $\mathrm{kg} \mathrm{hab}^{-1}$ ano $^{-1}$ e uma das principais causas do alto consumo de feijão é por ele ser uma fonte barata de proteína. Porém a produtividade do feijoeiro,

Trabalho financiado pelo Banco do Nordeste e CNPq

2 Mestrando em Produção Vegetal, Depto de Produção Vegetal, CCAUFES, Alegre - ES. E-mail: moraeswb@ hotmail.com

3 Acadêmico do curso de agronomia, Depto de Produção Vegetal, CCAUFES, Alegre - ES. E-mail: simonepaiva01@ hotmail. com,wan.b.m2@hotmail.com

4 Engo Agrônomo, Bolsista de PDJ do CNPq, Depto de Engenharia Rural, CCAUFES, Alegre - ES. giovanni@ cca.ufes.br

5 Engo Agrônomo, Prof. Adjunto Dr, DPI/UFV, Viçosa - MG. E-mail: smartins@dpi.ufv.br 
no Brasil, é considerada relativamente baixa quando comparada com os resultados obtidos sob condições experimentais. Várias são as sugestões atribuídas para esse resultado, tendo como principais fatores limitantes da produção vegetal a deficiência nutricional e o déficit hídrico, estes ocorrem em grandes extensões de áreas cultiváveis (Hungria et al., 2000; Nogueira et al., 2001).

Em várias situações de cultivo, a água tem se mostrado o recurso mais limitante ao crescimento e à produtividade das culturas. $\mathrm{O}$ volume explorado pelo sistema de raízes, a eficiência no uso da água e a capacidade de extração da água do solo determinam a capacidade competitiva de uma planta por esse recurso. Assim as características morfológicas e fisiológicas das plantas determinam suas habilidades competitivas pela água do solo (Procopio et al., 2004).

O rendimento das culturas depende da intensidade, duração e época de ocorrência do déficit hídrico, e da interação deste com outros fatores que determinam o rendimento final (Cunha \& Bergamaschi, 1992). O feijoeiro é considerado uma planta sensível ao estresse hídrico, principalmente em virtude da baixa capacidade de recuperação após a deficiência hídrica e sistema radicular pouco desenvolvido. A fase da planta mais sensível à deficiência de água é a reprodutiva, sendo altamente vulnerável desde o início da floração até o início da formação das vagens (Gomes et al., 2000). Esse efeito é causado principalmente pela baixa taxa de polinização e pelo aborto de óvulos, que causam abscisão dos órgãos reprodutivos (Kramer \& Boyer, 1995).

No entanto, como existe uma variabilidade na adaptação à seca entre espécies e dentro da espécie, deve-se avaliar o comportamento de diferentes materiais genéticos, em condições de seca, para a recomendação de cultivo, beneficiando assim, o melhoramento genético com o objetivo de se obter materiais com tolerância a condições de seca (Blum, 1997). Segundo Salisbury E Ross (1991) a produtividade é considerada uma das características mais intimamente relacionada com a água disponível para as plantas do que qualquer outro fator ambiental. Assim, a capacidade de as plantas se manterem túrgidas é tida como uma característica necessária para a garantia da produção, em locais onde ocorre o déficit hídrico.

O presente trabalho teve como objetivo avaliar linhagens promissoras de feijoeiro (Phaseolus vulgaris L.) tolerantes a seca, para inseri-las no programa de melhoramento genético do Estado do Espírito Santo.

\section{MATERIAL E MÈTODOS}

O experimento foi conduzido em casa de vegetação no campus do Centro de Ciências Agrárias da Universidade Federal do Espírito Santo (CCAUFES), Alegre - ES, com coordenadas geográficas de $20^{\circ} 45^{\prime}$ de latitude Sul, $41^{\circ} 28^{\prime}$ de longitude Oeste e altitude de $150 \mathrm{~m}$. Para a avaliação de linhagens promissoras tolerantes à seca, o plantio foi realizado em vasos de 10 L. Devido ao grande número de genótipos a serem avaliados o experimento foi realizado em duas fases. Na primeira fase foram utilizados 18 linhagens promissoras: IN 1, IN 2, IN 3, IN 4, IN 5, IN 6, IN 7, IN 8, IN 9, IN 10, IN 11, IN 12, IN 13 , IN 14, IN 15, IN 16, IN 17, IN 18 e 9 cultivares: EL 22, Bambuí, Pérola, Serrano, Xamego, Uirapuru, IAPAR 81, Vermelho, Capixaba Precoce e Brinco de Ouro, além de um padrão de tolerância (BATT 477) e outro de susceptibilidade ao estresse hídrico (Carioca), os quais foram inoculados com a estirpe padrão de Rhizobium (CIAT 899). Na segunda fase do experimento foram utilizados 16 genótipos locais pertencentes aos agricultores da comunidade agrícola da Fortaleza, situada no município de Muqui, ES: Imperial, Rio Doce, Baetão, Bate Estrada, Caeté Pé Curto, Iconha, Morgado, Mulatinho, Amarelinho, Vagem Riscada, Rosinha, Terrinha 1, Macuquinho, Mamona, Levanta Hipoteca, Amendoim Riscado, os quais foram, também, inoculados com a estirpe padrão de Rhizobium (CIAT 899).

O delineamento utilizado foi o inteiramente casualizados no esquema de parcelas subdivididas (nas parcelas foi utilizado o sistema de cultivo, com ou sem estresse hídrico, e nas subparcelas os genótipos), com três repetições e a unidade experimental foi um vaso com 2 plantas.

A acidez do solo foi corrigida com aplicação de calcário dolomítico e em cada vaso foi aplicada uma adubação mineral na forma de 04-14-08. As plantas foram irrigadas diariamente de forma a manter o solo sempre próximo à capacidade de campo. Nas parcelas que foram submetidas ao estresse hídrico, após 30 dias da emergência, período de pré-floração -estádio de formação dos botões florais- a irrigação foi interrompida por 15 dias.

Em cada rega, a correção da umidade do solo foi feita com base na diferença entre o peso atual 
do vaso e o peso prefixado para cada tratamento. Assumindo-se que a água da torneira apresentou relação peso/volume de 1:1, a irrigação foi realizada de modo a complementar a água faltante em termos de volume $(\mathrm{mL})$.

A umidade do solo no período de estresse hídrico foi determinada num intervalo de dois dias, pelo método EDABO (Evaporação Direta da Água em Banho de Óleo). No método utilizado, foram coletadas amostras do solo retiradas aleatoriamente em vários vasos nos quais tiveram pré-floração no mesmo dia, na profundidade do sistema radicular da cultura. Dessas amostras, foi feita uma amostra composta com peso médio de 100 gramas, que posteriormente foi submetida à fervura em óleo a temperatura de $190^{\circ} \mathrm{C}$ até que parasse o borbulhamento. Por meio de equivalência de peso, determinou-se a quantidade de água evaporada em 2 dias. A umidade do vaso foi calculada utilizando-se a seguinte fórmula:

$$
\text { Unidade }(\%)=\frac{100 \times \text { Vol. Evaporado }}{100-\text { Vol. Evaporado }}
$$

A umidade do solo nos vasos sob estresse hídrico foi mantida próxima ao ponto de murchamento, com um fator de disponibilidade de água (f) entre 0,3 a 0,4. Após o período de estresse hídrico de 15 dias, as parcelas foram irrigadas normalmente até o final do ciclo da cultura.

No estádio de maturação foram avaliadas as seguintes características: número de nós, altura da planta, número de vagens por planta, número de sementes por vagem, peso médio de 100 sementes e produtividade total. Os dados foram analisados por meio de análise de variância e de teste de média.

\section{RESULTADOS E DISCUSSÃO}

As características número de vagens (NV), número de sementes por vagem (NSV), peso médio de cem sementes (PMCS) e produtividade em gramas por planta (PROD) de genótipos de feijoeiro cultivados com e sem estresse hídrico, mostrou-se significativa a $5 \%$ de probabilidade para a interação sistema de cultivo $\mathrm{x}$ genótipo.

De acordo com os resultados expressos na Tabela 1 observou-se que houve diferença significativa no número de vagens (NV), entre os genótipos nos sistemas de cultivo. Os genótipos IN
3, IN 4, IN 5, IN 6, IN 7, IN 8, IN 11, IN 13, IN 18, Carioca, Bambuí, Pérola, Xamego e Uirapuru demonstraram-se superiores quando comparados entre os dois sistemas, os demais genótipos não apresentaram diferença significativa entre os sistemas de cultivo, mostrando que estes genótipos são tolerantes ao estresse hídrico. Na comparação dentro do sistema de cultivo sem estresse, o teste estatístico apresentou diferença significativa nos genótipos IN 3, IN 4, IN 5, IN 6, IN 7, IN 8, IN 9, IN 10, IN 11, IN 13, IN 15, IN 16, IN18, BAT 477, EL22, Capixaba precoce, Carioca, Bambuí, Pérola, Serrano, Xamego; Uirapuru e Brinco de ouro, sendo superiores aos demais. No sistema de cultivo com estresse hídrico, os genótipos IN 15, EL 22, Capixaba precoce e Serrano foram superiores; os demais genótipos não apresentaram diferença significativa neste sistema de cultivo. Confirmando os dados de Guimarães (1996) o número de vagens, quando comparados os sistemas de cultivo sem e com estresse, este último ocasionou uma redução desta característica em quase a metade dos genótipos avaliados.

Analisando a variável número de sementes por vagem (NSV) na Tabela 1 podemos analisar que entre os sistemas de cultivo, os genótipos IN 6, IN 12, Pérola, Serrano e Uirapuru tiveram maior quantidade de sementes por vagem no sistema sem estresse. Os demais genótipos não apresentaram diferença entre si. Dentro do sistema de cultivo sem estresse, os genótipos IN 5, IN 13, BATT 477, Bambuí, Serrano, Xamego e Uirapuru foram superiores, não apresentando diferença significativa entre os demais. Dentro do sistema de cultivo com estresse, os genótipos que se diferenciaram com maior quantidade de número de sementes por vagem foram IN 5 e Xamego. O número de sementes por vagem, no sistema de cultivo com estresse, também diminuiu nos genótipos IN 6, IN 12, Pérola, Serrano e Uirapuru, mostrando situações encontradas em estudos realizados por Fageria et al. (1991) e Guimarães (1996).

Para a característica peso médio de cem sementes, os genótipos IN 4, IN6, IN 10, IN12, Carioca e Pérola se diferenciaram estatisticamente quando comparados entre os sistemas de cultivo, sendo as sementes com maior peso aquelas oriundas das plantas do sistema de cultivo sem estresse. Dentro do sistema de cultivo sem estresse os genótipos IN 8 E IN 12 apresentaram as maiores médias. Dentro do sistema de cultivo com estresse, com exceção 
Tabela 1

Número de vagens (NV), número de sementes por vagem (NSV), peso médio de cem sementes (PMCS) e produtividade em gramas por planta (PROD) de genótipos de feijão cultivados com e sem estresse hídrico

\begin{tabular}{|c|c|c|c|c|c|c|c|c|}
\hline \multirow{3}{*}{ GENÓTIPOS } & \multicolumn{2}{|c|}{ NV } & \multicolumn{2}{|c|}{ NSV } & \multicolumn{2}{|c|}{ PMCS } & \multicolumn{2}{|c|}{ PROD } \\
\hline & SEM & COM & SEM & COM & SEM & COM & SEM & COM \\
\hline & Estresse & Estresse & Estresse & Estresse & Estresse & Estresse & Estresse & Estresse \\
\hline IN 1 & & - & - & - & - & - & - & - \\
\hline IN 2 & $1,00 \mathrm{cA}^{1}$ & $0,67 \mathrm{bA}$ & $2,17 \mathrm{bA}$ & $1,00 \mathrm{dA}$ & $23,55 \mathrm{cA}$ & $29,13 \mathrm{aA}$ & $0,97 \mathrm{dA}$ & $0,43 \mathrm{aA}$ \\
\hline IN 3 & $6,67 \mathrm{aA}$ & $3,00 \mathrm{bB}$ & $3,16 \mathrm{bA}$ & $2,22 \mathrm{cA}$ & $24,70 \mathrm{cA}$ & $13,89 \mathrm{aA}$ & $4,93 \mathrm{bA}$ & $0,96 \mathrm{aB}$ \\
\hline IN 4 & $6,33 \mathrm{aA}$ & $2,33 \mathrm{bB}$ & $2,70 \mathrm{bA}$ & $2,00 \mathrm{cA}$ & $29,09 \mathrm{bA}$ & $16,86 \mathrm{aB}$ & $4,70 \mathrm{bA}$ & $0,83 \mathrm{aB}$ \\
\hline IN 5 & $8,00 \mathrm{aA}$ & $2,33 \mathrm{bB}$ & $4,65 \mathrm{aA}$ & $4,33 \mathrm{aA}$ & $25,34 \mathrm{cA}$ & $20,67 \mathrm{aA}$ & $9,40 \mathrm{aA}$ & $1,94 \mathrm{aB}$ \\
\hline IN 6 & $4,67 \mathrm{aA}$ & $1,00 \mathrm{bB}$ & $2,43 \mathrm{bA}$ & $1,00 \mathrm{~dB}$ & $31,75 \mathrm{bA}$ & $16,32 \mathrm{aB}$ & $3,51 \mathrm{cA}$ & $0,33 \mathrm{aB}$ \\
\hline IN 7 & $8,00 \mathrm{aA}$ & $3,67 \mathrm{bB}$ & $2,80 \mathrm{bA}$ & $2,17 \mathrm{cA}$ & $25,73 \mathrm{cA}$ & $16,22 \mathrm{aA}$ & $5,60 \mathrm{bA}$ & $1,48 \mathrm{aB}$ \\
\hline IN 8 & $8,00 \mathrm{aA}$ & $3,67 \mathrm{bB}$ & $2,48 \mathrm{bA}$ & $1,83 \mathrm{cA}$ & $43,76 \mathrm{aA}$ & $32,35 \mathrm{aA}$ & $8,62 \mathrm{aA}$ & $2,25 \mathrm{aB}$ \\
\hline IN 9 & $5,67 \mathrm{aA}$ & $3,33 \mathrm{bA}$ & $2,83 \mathrm{bA}$ & $1,89 \mathrm{cA}$ & $20,00 \mathrm{cA}$ & $15,75 \mathrm{aA}$ & $3,25 \mathrm{cA}$ & $1,10 \mathrm{aA}$ \\
\hline IN 10 & $6,00 \mathrm{aA}$ & $3,33 \mathrm{bA}$ & $2,33 \mathrm{bA}$ & $2,14 \mathrm{cA}$ & $30,56 \mathrm{bA}$ & $11,14 \mathrm{aB}$ & $4,71 \mathrm{bA}$ & $0,81 \mathrm{aB}$ \\
\hline IN 11 & $9,67 \mathrm{aA}$ & $2,67 \mathrm{bB}$ & $2,86 \mathrm{bA}$ & $1,50 \mathrm{cA}$ & $20,25 \mathrm{cA}$ & $12,22 \mathrm{aA}$ & $5,44 \mathrm{bA}$ & $0,48 \mathrm{aB}$ \\
\hline IN 12 & $2,00 \mathrm{bA}$ & $0,00 \mathrm{bA}$ & $1,83 \mathrm{bA}$ & $0,00 \mathrm{~dB}$ & $39,51 \mathrm{aA}$ & $0,00 \mathrm{bB}$ & $1,38 \mathrm{dA}$ & $0,00 \mathrm{aA}$ \\
\hline IN 13 & $8,33 \mathrm{aA}$ & $2,00 \mathrm{bB}$ & $3,67 \mathrm{aA}$ & $3,28 \mathrm{bA}$ & $26,83 \mathrm{bA}$ & $23,50 \mathrm{aA}$ & $8,17 \mathrm{aA}$ & $1,55 \mathrm{aB}$ \\
\hline IN 14 & - & - & - & - & - & - & - & - \\
\hline IN 15 & $10,00 \mathrm{aA}$ & $6,67 \mathrm{aA}$ & $3,29 \mathrm{bA}$ & $2,91 \mathrm{bA}$ & $23,00 \mathrm{cA}$ & $18,04 \mathrm{aA}$ & $7,45 \mathrm{aA}$ & $3,13 \mathrm{aB}$ \\
\hline IN 16 & $5,67 \mathrm{aA}$ & $3,33 \mathrm{bA}$ & $1,85 \mathrm{bA}$ & $2,80 \mathrm{bA}$ & $16,80 \mathrm{cA}$ & $14,56 \mathrm{aA}$ & $3,87 \mathrm{cA}$ & $1,37 \mathrm{aA}$ \\
\hline IN 17 & - & - & - & - & - & - & - & - \\
\hline IN 18 & $6,33 \mathrm{aA}$ & $2,33 \mathrm{bB}$ & $2,59 \mathrm{bA}$ & $2,67 \mathrm{bA}$ & $19,13 \mathrm{cA}$ & $15,14 \mathrm{aA}$ & $3,13 \mathrm{cA}$ & $1,01 \mathrm{aA}$ \\
\hline BAT 477 & $5,67 \mathrm{aA}$ & $3,00 \mathrm{bA}$ & $4,44 \mathrm{aA}$ & $3,42 \mathrm{bA}$ & $24,37 \mathrm{cA}$ & $14,90 \mathrm{aA}$ & $5,95 \mathrm{bA}$ & $1,57 \mathrm{aB}$ \\
\hline EL 22 & $6,33 \mathrm{aA}$ & $8,00 \mathrm{aA}$ & $3,25 \mathrm{bA}$ & $3,19 \mathrm{bA}$ & $18,67 \mathrm{cA}$ & $21,23 \mathrm{aA}$ & $4,71 \mathrm{bA}$ & $5,38 \mathrm{aA}$ \\
\hline Cap. Precoce & $7,00 \mathrm{aA}$ & $5,00 \mathrm{aA}$ & $2,21 \mathrm{bA}$ & $3,29 \mathrm{bA}$ & $16,37 \mathrm{cA}$ & $16,26 \mathrm{aA}$ & $5,66 \mathrm{bA}$ & $2,71 \mathrm{aB}$ \\
\hline Carioca & $7,33 \mathrm{aA}$ & $2,33 \mathrm{bB}$ & $2,79 \mathrm{bA}$ & $2,61 \mathrm{bA}$ & $28,31 \mathrm{bA}$ & $11,42 \mathrm{aB}$ & $6,26 \mathrm{bA}$ & $0,59 \mathrm{aB}$ \\
\hline Bambuí & $5,33 \mathrm{aA}$ & $1,67 \mathrm{bB}$ & $4,02 \mathrm{aA}$ & $3,50 \mathrm{bA}$ & $25,72 \mathrm{cA}$ & $14,73 \mathrm{aA}$ & $5,46 \mathrm{bA}$ & $0,85 \mathrm{aB}$ \\
\hline Pérola & 7,33aA & $1,00 \mathrm{bB}$ & $2,78 \mathrm{bA}$ & $0,50 \mathrm{~dB}$ & $29,71 \mathrm{bA}$ & $4,43 \mathrm{bB}$ & $5,84 \mathrm{bA}$ & $0,13 \mathrm{aB}$ \\
\hline Serrano & $5,67 \mathrm{aA}$ & $8,33 \mathrm{aA}$ & $4,86 \mathrm{aA}$ & $2,72 \mathrm{bB}$ & $27,60 \mathrm{bA}$ & $19,44 \mathrm{aA}$ & $7,27 \mathrm{aA}$ & $3,16 \mathrm{aB}$ \\
\hline Xamego & $8,00 \mathrm{aA}$ & $2,00 \mathrm{bB}$ & $3,85 \mathrm{aA}$ & $5,06 \mathrm{aA}$ & $21,88 \mathrm{cA}$ & $13,01 \mathrm{aA}$ & $6,65 \mathrm{bA}$ & $1,35 \mathrm{aB}$ \\
\hline Uirapuru & $7,33 \mathrm{aA}$ & $2,00 \mathrm{bB}$ & $4,19 \mathrm{aA}$ & $1,94 \mathrm{cB}$ & $25,45 \mathrm{cA}$ & $16,15 \mathrm{aA}$ & $7,83 \mathrm{aA}$ & $0,62 \mathrm{aB}$ \\
\hline IAPAR 81 & $4,00 \mathrm{bA}$ & $2,67 \mathrm{bA}$ & $2,03 \mathrm{bA}$ & $1,83 \mathrm{cA}$ & $28,20 \mathrm{bA}$ & $19,59 \mathrm{aA}$ & $2,29 \mathrm{cA}$ & $0,95 \mathrm{aA}$ \\
\hline Vermelho & $3,67 \mathrm{bA}$ & $2,67 \mathrm{bA}$ & $2,26 \mathrm{bA}$ & $1,39 \mathrm{cA}$ & $15,16 \mathrm{cA}$ & $8,21 \mathrm{bA}$ & $2,46 \mathrm{cA}$ & $0,41 \mathrm{aA}$ \\
\hline Brinco de ouro & $5,33 \mathrm{aA}$ & $3,00 \mathrm{bA}$ & $2,14 \mathrm{bA}$ & $1,67 \mathrm{cA}$ & $15,05 \mathrm{cA}$ & $18,00 \mathrm{aA}$ & $1,75 \mathrm{dA}$ & $1,10 \mathrm{aA}$ \\
\hline
\end{tabular}

1 Médias seguidas pela mesma letra minúscula na coluna e mesma letra maiúscula na linha pertencem ao mesmo agrupamento, ao nível de 5\% de significância, pelo teste de Scott-Knott. 
dos genótipos Pérola e Vermelho, que apresentaram menores médias, não houve diferença significativa entre os demais. No peso médio de cem sementes, os genótipos IN 2, EL 22 e Brinco de ouro tiveram um aumento significativo, devido, provavelmente, pela concentração de reserva em apenas algumas sementes.

Para a variável produtividade a comparação entre os sistemas de cultivo mostra que houve diferença estatística significativa para os genótipos IN 3, IN 4, IN 5, IN 6, IN 7, IN 8, IN 10, IN 11, IN 13IN 15, BATT 477, Capixaba precoce, Carioca, Bambuí, Pérola, Serrano, Xamego e Uirapuru, sendo os mais produtivos, já os demais genótipos não apresentaram diferença significativa. Dentro do sistema de cultivo sem estresse destacou-se os genótipos IN 5, IN 8, IN 13, IN 15, Serrano e Uirapuru, entretanto, dentro do sistema de cultivo com estresse não houve diferença significativa entre os genótipos.

De forma geral estudos realizados por Norman et al. (1995) mostram que situações de estresses principalmente durante o período de pré-floração, causam redução no número de vagens e grãos, refletindo diretamente numa menor produtividade.

Na segunda fase deste experimento verificou-se que a interação sistema de cultivo $\mathrm{x}$ genótipo foi significativa apenas para o número de flores. $\mathrm{Na}$ Tabela 2 está apresentado o estudo da interação sistema de cultivo $\mathrm{x}$ genótipos para o número de flores, na qual pode se notar a diferença significativa entre os sistemas de cultivo apenas para os genótipos Caeté pé Curto, Iconha, Vagem Riscada e Macuquinho, sendo estes suscetíveis ao déficit hídrico, pois o menor número de flores acarreta menor número de vagens e conseqüentemente menor produtividade (Kramer \& Boyer, 1995). Para os demais genótipos não houve diferença significativa entre os sistemas de cultivo, mostrando que estes genótipos, para esta característica, se comportam de maneira semelhante, tanto na presença quanto na ausência do estresse hídrico.

Na Tabela 3 está apresentado o estudo entre os sistemas de cultivo para as carcterísticas: número

Tabela 2

Número de flores de genótipos de feijoeiro cultivados sem e com déficit hídrico

\begin{tabular}{|l|c|c|}
\hline \multirow{2}{*}{ Genótipos } & \multicolumn{2}{c|}{ Flor } \\
\cline { 2 - 3 } & Sem Déficit & Com Déficit \\
\hline Imperial & $4,33 \mathrm{bA}^{1}$ & $12,33 \mathrm{aA}$ \\
\hline Rio Doce & $7,67 \mathrm{bA}$ & $7,67 \mathrm{aA}$ \\
\hline Baetão & $5,00 \mathrm{bA}$ & $1,67 \mathrm{aA}$ \\
\hline Bate Estrada & $7,33 \mathrm{bA}$ & $4,67 \mathrm{aA}$ \\
\hline Caeté Pé Curto & $13,00 \mathrm{aA}$ & $3,67 \mathrm{aB}$ \\
\hline Iconha & $22,00 \mathrm{aA}$ & $5,33 \mathrm{aB}$ \\
\hline Morgado & $8,67 \mathrm{bA}$ & $8,00 \mathrm{aA}$ \\
\hline Mulatinho & $6,00 \mathrm{bA}$ & $1,67 \mathrm{aA}$ \\
\hline Amarelinho & $11,33 \mathrm{bA}$ & $10,67 \mathrm{aA}$ \\
\hline Vagem Riscada & $12,67 \mathrm{aA}$ & $2,00 \mathrm{aB}$ \\
\hline Rosinha & $4,67 \mathrm{bA}$ & $4,00 \mathrm{aA}$ \\
\hline Terrinha 1 & $6,67 \mathrm{bA}$ & $3,33 \mathrm{aA}$ \\
\hline Macuquinho & $17,67 \mathrm{aA}$ & $6,00 \mathrm{aB}$ \\
\hline Mamona & $8,67 \mathrm{bA}$ & $6,00 \mathrm{aA}$ \\
\hline Levanta Hipoteca & $9,00 \mathrm{bA}$ & $0,67 \mathrm{aA}$ \\
\hline Amendoim Riscado & $8,67 \mathrm{bA}$ & $6,00 \mathrm{aA}$ \\
\hline
\end{tabular}

1 Médias seguidas pela mesma letra minúscula nas colunas e maiúscula nas linhas pertencem ao mesmo agrupamento, ao nível de 5\% de significância, pelo teste de Scott-Knott.

Tabela 3

Médias do número de nós (NOS), altura de plantas (ALT), número de vagens (NV), número de sementes (NS), peso de sementes (PS) e peso de cem sementes (PCS) para plantas de feijão cultivadas sem e com déficit hídrico

\begin{tabular}{|l|c|c|c|c|c|c|}
\hline \multicolumn{1}{|c|}{ Sistema de Cultivo } & NOS & ALT & NV & NS & PS & PCS \\
\hline Sem Déficit & $14,8438 \mathrm{a}^{1}$ & $105,0833^{2}$ & $1,7708 \mathrm{a}$ & $4,3542 \mathrm{a}$ & $0,8095 \mathrm{a}$ & $14,3425 \mathrm{a}$ \\
\hline Com Déficit & $12,8542 \mathrm{~b}$ & 100,7188 & $0,6042 \mathrm{~b}$ & $1,6250 \mathrm{~b}$ & $0,3200 \mathrm{~b}$ & $6,4172 \mathrm{~b}$ \\
\hline
\end{tabular}

1 Médias seguidas por letras diferentes na coluna não pertencem ao mesmo grupo, ao nível de 5\% de significância, pelo teste de Scott-Knott.

2 Não significativo ao nível de $5 \%$ de significância, pelo teste $\mathrm{F}$. 
de nós (NOS), altura de plantas (ALT), número de vagens (NV), número de sementes (NS), peso de sementes (PS) e peso de cem sementes (PCS) para plantas de feijão cultivadas sem e com déficit hídrico. Pode se verificar que todas as características foram superiores no sistema sem déficit hídrico, o que de certa forma já era esperado ocorrer, pois neste sistema as plantas foram irrigadas continuamente.

Na Tabela 4 está apresentado o estudo entre os genótipos. Pode ser constatado que entre todos os genótipos o "Levanta Hipoteca" apresentou maior número de nós e altura de planta, o que ratificou o seu hábito de crescimento indeterminado.

Para as características ligadas a produção: número de vagens, número de sementes e peso de sementes por planta pode ser observada (Tabela 4), de forma geral, a superioridade dos genótipos Imperial, Bate Estrada, Morgado, Amarelinho, Vagem Riscada e Macuquinho. Pode ser destacado que os genótipos Vagem Riscada e Macuquinho foram suscetíveis ao déficit hídrico, o qual fica implícito a importância destes materiais serem incluídos em um programa de melhoramento que tem em vista a seleção para maior tolerância ao estresse hídrico, uma vez que estes genótipos pertencem aos agricultores da comunidade da Fortaleza e não passaram por nenhum processo de melhoramento genético.

Concordando com Pimentel et al., (2000) a produtividade do feijoeiro sob estresse hídrico diminui e as reduções nas características avaliadas podem ser justificadas pela condição de baixa umidade o qual foi submetido o feijoeiro no período crítico (pré-floração e enchimento dos botões florais).

\section{CONCLUSÕES}

Em termos de produtividade pode-se notar que a diferença não-significativa entre os sistemas de

Tabela 4

Médias do número de nós (NOS), altura de plantas (ALT), número de vagens (NV), número de sementes (NS), peso de sementes (PS) e peso de cem sementes (PCS) para diferentes genótipos de feijoeiro

\begin{tabular}{|c|c|c|c|c|c|c|}
\hline GENÓTIPOS & NOS & ALT & NV & NS & PS & PCS \\
\hline Imperial & $16,83 b^{1}$ & $107,83 b$ & $1,67 \mathrm{a}$ & $5,50 \mathrm{a}$ & $1,21 \mathrm{a}$ & $13,60^{2}$ \\
\hline Rio Doce & $14,67 b$ & $95,50 \mathrm{~b}$ & $1,00 \mathrm{~b}$ & $2,33 b$ & $0,46 b$ & 10,73 \\
\hline Baetão & $15,25 b$ & $92,00 \mathrm{~b}$ & $1,00 \mathrm{~b}$ & $2,83 b$ & $0,47 b$ & 10,90 \\
\hline Bate Estrada & $13,50 \mathrm{~b}$ & $115,00 \mathrm{~b}$ & $1,33 b$ & $4,67 \mathrm{a}$ & $1,13 \mathrm{a}$ & 16,84 \\
\hline Caeté Pé Curto & $13,92 b$ & $100,83 b$ & $0,67 b$ & $2,17 \mathrm{~b}$ & $0,42 b$ & 9,93 \\
\hline Iconha & $6,92 d$ & $61,83 \mathrm{c}$ & $0,50 \mathrm{~b}$ & $0,83 b$ & $0,22 b$ & 7,63 \\
\hline Morgado & $13,67 b$ & $88,00 \mathrm{~b}$ & $1,67 \mathrm{a}$ & $4,00 \mathrm{a}$ & $0,75 b$ & 10,97 \\
\hline Mulatinho & $9,83 \mathrm{c}$ & $57,08 \mathrm{c}$ & $1,17 \mathrm{~b}$ & $2,50 \mathrm{~b}$ & $0,30 \mathrm{~b}$ & 6,17 \\
\hline Amarelinho & $13,67 b$ & $96,25 b$ & $2,67 \mathrm{a}$ & $6,67 \mathrm{a}$ & $1,26 \mathrm{a}$ & 18,56 \\
\hline Vagem Riscada & $14,83 b$ & $102,58 b$ & $1,67 \mathrm{a}$ & $4,17 \mathrm{a}$ & $0,57 \mathrm{~b}$ & 4,62 \\
\hline Rosinha & $11,75 \mathrm{c}$ & $129,08 b$ & $0,50 \mathrm{~b}$ & $1,00 \mathrm{~b}$ & $0,23 b$ & 4,20 \\
\hline Terrinha 1 & $14,92 b$ & $95,17 \mathrm{~b}$ & $0,67 b$ & $0,83 b$ & $0,26 \mathrm{~b}$ & 20,83 \\
\hline Macuquinho & $12,25 \mathrm{c}$ & $112,83 b$ & $3,00 \mathrm{a}$ & $7,50 \mathrm{a}$ & $1,26 \mathrm{a}$ & 12,28 \\
\hline Mamona & $15,50 \mathrm{~b}$ & $104,00 \mathrm{~b}$ & $1,17 \mathrm{~b}$ & $2,50 \mathrm{~b}$ & $0,53 b$ & 11,54 \\
\hline Levanta Hipoteca & $21,50 \mathrm{a}$ & $207,00 \mathrm{a}$ & $0,00 \mathrm{~b}$ & $0,00 \mathrm{~b}$ & $0,00 \mathrm{~b}$ & 0,00 \\
\hline Amendoim Riscado & $12,58 \mathrm{c}$ & $81,42 \mathrm{c}$ & $0,33 b$ & $0,33 b$ & $0,06 \mathrm{~b}$ & 5,67 \\
\hline
\end{tabular}

1 Médias seguidas pela mesma letra na coluna pertencem ao mesmo grupo, ao nível de 5\% de significância, pelo teste de Scott-Knott;

2 Não significativo ao nível de $5 \%$ de significância, pelo teste F. 
cultivo sem e com estresse para os genótipos IN 2, IN 9, IN 12, IN 14, IN 16, IN 17, IN 18, EL-22, IAPAR 81, Vermelho e Brinco de Ouro, mostrou que estes genótipos são tolerantes ao estresse hídrico. Porém estes materiais na maioria são pouco produtivos. Salienta-se, no entanto, que a maioria destes materiais são linhagens em desenvolvimentos, necessitando de maiores estudos em um programa

\section{LITERATURA CITADA}

BLUM, A. 1997. Crop responses to drought and the interpretation of adaptation. In: BELHASSEN, E. (Ed.). Drought tolerance in higher plants. Genetical, physiological and molecular biological analysis. Dordrecht: Kluwer Academic, p. 57-70.

CUNHA, G.R.; BERGAMASCHI, H. 1992. Efeito da disponibilidade hídrica sobre o rendimento das culturas. In: BERGAMASCHI, H. (Coord.). Agrometeorologia aplicada à irrigação. Porto Alegre: UFRGS-Ed. Universitária, p. 85-97.

FAGERIA, N.K; BALIGAR, V.C.; JONES, C.A. 1991. Common bean and cowpea. In: FAGERIA, N.K; BALIGAR, V.C.; JONES, C.A. (Eds.). Growth and mineral nutrition of field crops. New York: Maecel Dekker, p. 280-318.

GOMES, A.A.; ARAUJO, A.P.; ROSSIELLO, R.O.P.; PIMENTEL, C. 2000. Acumulação de biomassa, características fisiológicas e rendimento de grãos em cultivares de feijoeiro irrigado e sob sequeiro. Pesquisa agropecuária brasileira, Brasilia, vol. 35, n 10, p. 1927-1937, out.

GUIMARÃES, C.M. 1996. Relações hódricas. In: ARAÚJO, R.S.; RAVA, C.A; STONE, L.F; ZIMMERMANN, M.J. DE O. (Eds.). Cultura do feijoeiro comun no Brasil. Piracicaba: Potafos, p. 139-168.

HUNGRIA, M.; ANDRADE, D.S.; CHUEIRE, L.A.O.; PROBANZA, A.; GUTTIERREZ-MANERO, F.J.; MEGIAS, M. 2000. Isolation and characterization of new efficient na competitive bean (Phaseolus vulgaris de melhoramento e que poderão vir a ter maiores produtividades.

\section{AGRADECIMENTOS}

Ao Banco do Nordeste pela colaboração financeira e ao CNPq pelas bolsas de Iniciação Científica e de Pós-doutorado.

L.) rhizobia from Brazil. Soil Biology \& Biochemistry, v. 32 , p. $1515-1528$.

KRAMER, P.J.; BOYER, J.S. 1995. Water relations of plants and soils. San Diego: Academic, 495 p.

NOGUEIRA, R.J.M.C.; MORAES, J.A.P.V.; BURITY, H.A. 2001. Alterações na resistência à difusão de vapor das folhas e relações hídricas em aceroleira submetidas a déficit de água. Revista Brasileira de Fisiologia Vegetal, Londrina, V. $13, n^{\circ} 1$, p. $75-87$.

NORMAN, M.J.T.; PEARSON, C.J.; SEARLE, P.G.E. 1995. Common bean (Phaseolus vulgaris L.). In: NORMAN, M.J.T.; PEARSON, C.J.; SEARLE, P.G.E. (Eds.). The ecology of tropical food crops. Cambridge: Cambridge University Press, p. 208-224.

PIMENTEL, C.; PÉREZ, A.J.C. 2000. Estabelecimento de parâmetros para avaliação de tolerância à seca, em genótipos de feijoeiro. Pesquisa Agropecuária Brasileira, vol. 35, nº 1 , Jan. 2000.

PROCOPIO, S.O.; SANTOS, J.B.; SILVA, A.A.; ET AL., 2004. Ponto de murcha permanente de soja, feijão e plantas daninhas. Planta daninha, jan./mar. 2004, vol. 22, no 1 , p. 35-41. ISSN 0100-8358.

SALISBURY, F.B.; ROSS, C.W. 1991. Plant physiology. 4 ed., California: Wadsworth Publishing Company, 682 p.

YOKOYAMA, L.P. 2002. O feijão no Brasil no período de 1984/5 a 1999/00: aspectos conjunturais. In: CONGRESSO NACIONAL DE PESQUISA DE FEIJÃO, 7, 2002, Viçosa. Anais... Viçosa: DFT, p. 654-657. 
